\title{
Intenção de mudança de comportamento em adolescentes para a prática de atividades físicas de lazer
}

CDD. 20.ed. 790.1

796.011
Marcos Gonçalves MACIEL* Ricardo Teixeira VEIGA**

\section{Resumo}

0 objetivo deste estudo foi investigar a intenção da mudança de comportamento de adolescentes de diferentes níveis socioeconômicos (NSE) para a prática de atividades físicas de lazer, adotando o Modelo Transteorético e a Teoria do Comportamento Planejado (TCP). Foi realizado um estudo transversal com a participação de 416 adolescentes, pertencentes a escolas do ensino médio nas cidades de Belo Horizonte e Contagem/MG, com média de idade de 16,4 $\pm 1,2$ anos. As análises foram feitas utilizando estatísticas descritivas e paramétricas (ANOVA e regressão logística). Os resultados encontrados demonstraram que a maioria dos adolescentes é inativa no lazer, e às variáveis, sexo, atitude e controle percebido são os fatores mais relevantes para classificar o nível de atividade física de lazer, sendo que as variáveis sociais (nivel socioeconômico e norma subjetiva) têm pouca importância para predizer a intenção de mudança de comportamento para a prática dessas atividades.

UnITERMos: Atividade física de lazer; Estágio de mudança de comportamento; Teoria do comportamento planejado.

\section{Introdução}

Segundo a World Health Organization (2004), aproximadamente $70 \%$ da população mundial pode ser considerada fisicamente inativa no lazer. Este fenômeno na atualidade pode ser atribuído a diversos fatores como o crescimento da insegurança e do esvaziamento dos espaços públicos nos centros urbanos; a carência de espaços e equipamentos de lazer comunitários que permitem a prática de atividades físicas; o desenvolvimento tecnológico; a falta de recursos financeiros pessoais para pagar um lugar que ofereça segurança, conforto e orientação profissional adequada; falta de tempo decorrente do ritmo de vida moderno, dentre outros fatores (CAMŌES \& LOPES, 2008; MaCiel, 2009; NAHAS, 2006).

Nos últimos anos algumas teorias comportamentais vêm sendo utilizadas para compreender a adesão às práticas de atividades físicas de lazer. As teorias mais empregadas para estudar esse fenômeno são: 1) Modelo da Crença em Saúde; 2) Teoria Cognitiva Social; 3) Modelo Ecológico; 4) Teoria do Comportamento Planejado (TCP); 5) Modelo Transteorético (MT) (Bauman, Sallis, Dzewaltowski \& Oewn, 2002; Biddle \& NigG, 200; Dumith, 2008; Seabra, Thomis,
ANJos \& MAIA, 2008). Neste artigo, propomos associar o MT à TCP, visando conhecer os antecedentes atitudinais dos estágios de mudança comportamental relacionados à prática dessas atividades.

O MT elaborado por Prochaska e MARCus (1994), surgiu a partir do modelo original proposto por PROCHASKa e DiClemente (1983), em que esses autores elaboraram a teoria baseada nos constructos de estágios de mudança do comportamento (EMC), e dos processos de mudança do comportamento. Este trabalho abordará somente as características dos EMC. Esses podem ser caracterizados como: 1) Pré-contemplação - $\mathrm{O}$ indivíduo não tem a intenção de mudar o seu comportamento nos próximos seis meses; Contemplação - $\mathrm{O}$ indivíduo tem a intenção de mudar o seu comportamento nos próximos seis meses; 3 ) Preparação - $\mathrm{O}$ indivíduo pretende agir num futuro próximo (em geral no próximo mês); 4) Ação - O comportamento já foi incorporado por menos de seis meses; 5) A ação já acontece a mais de seis meses e as chances do retorno ao antigo comportamento são menores.

A TCP preconizada por AJZEN (1985) é uma das teorias da ação mais utilizadas para investigar o 
comportamento humano (BAGOZZI, GÜRHAN-CANLI \& Priester, 2002). Esta teoria tem como objetivo explicar e predizer comportamentos sociais, ou seja, busca prever as intenções comportamentais, que precedem e condicionam os comportamentos reais, em vez de conhecer unicamente as atitudes e crenças do indivíduo. Por intenção comportamental, AJZEN (1985), refere-se à disposição ou planejamento de uma pessoa de executar ou não um comportamento.

Em síntese, segundo AJZEN (2002), o comportamento humano é guiado por três tipos de crenças: 1) Comportamentais - sobre as consequências prováveis de um comportamento; 2) Normativas - sobre as expectativas normativas de terceiros; 3) Controle percebido - crenças a respeito da presença de fatores que podem impedir ou facilitar a performance de um comportamento.

A TCP assume que crenças são proposições sobre relações entre dois objetos concretos ou abstratos ou entre um objeto e seus atributos (KRÜGER, 1986). Atitude é um constructo unidimensional, de natureza afetiva e avaliativa, associado a uma apreciação positiva ou negativa de um objeto social (AJZEN \& FISHBEIN, 1980). Norma subjetiva é a percepção de pressão social exercida por pessoas importantes em relação à realização ou não de um comportamento. Intenções comportamentais se referem à disposição ou planejamento de executar um comportamento, por exemplo, a intenção de comprar um produto ou de devolvê-lo à loja.

Em seus respectivos agregados, crenças comportamentais produzem uma atitude (des)favorável em relação ao comportamento; crenças normativas resultam em pressão social perceptível ou norma subjetiva, e crenças de controle podem facilitar ou impedir a performance de um comportamento. Em combinação, atitude em relação ao comportamento,

\section{Método}

Esta pesquisa caracteriza-se como transversal descritiva com amostragem intencional não probabilística. O estudo foi aprovado pelo Comitê de Ética e Pesquisa da UFMG (protocolo de no 554/2008). Todos os participantes assinaram o termo de consentimento livre e esclarecido; os menores de 18 anos tiveram a autorização de seus responsáveis, que também assinaram o referido termo de consentimento.

Participaram adolescentes de ambos os sexos com idade compreendida entre 14 e 19 anos e regularmente matriculados no ensino médio em escolas pertencentes à região Metropolitana de Belo norma subjetiva e percepção se o comportamento pode ou não ser controlado conduz a formação de uma intenção comportamental.

Como regra geral, segundo Ajzen, quanto mais favorável for a atitude, norma subjetiva e controle percebido, maior deveria ser a intenção pessoal de realizar o comportamento. Finalmente, dado um suficiente grau de controle do comportamento, as pessoas tendem a realizar suas intençōes quando as oportunidades aparecem. Considerando esses aspectos, a intenção comportamental é considerada o antecessor imediato do comportamento, como apresentado em diversos estudos (Armitage, 2005; Blanchard, Fischer, SPARling, NeHL, RhODES, COURNEYA \& BAKER, 2008; Chatzisarantis, Hagger \& Brickell, 2008; Cheung, 2007; Rhodes, Blanchard, Courneya \& Plotnikoff, 2007; Zhang, MiddlestadT \& Ji, 2007).

Em virtude das elevadas taxas de inatividade física no lazer, não obstante toda a informação científica disponível e divulgada pelos meios de comunicação e profissionais da área da saúde sobre os seus efeitos deletérios da inatividade física pode-se fazer alguns questionamentos: Quais seriam os fatores mais relevantes para a intenção da mudança de comportamento para a prática de atividades físicas de lazer? Quais as crenças comportamentais que as pessoas mais valorizam, ou há uma associação entre as mesmas? Esses fatores variam quando se compara o sexo e o nível socioeconômico (NSE) diferentes entre si nessa faixa etária? Considerando os aspectos descritos, o objetivo desse trabalho é investigar a intenção de mudança de comportamento de adolescentes de diferentes níveis NSE em cada estágio de mudança proposto pelo MT utilizando os constructos da TCP.
Horizonte/MG. Foram selecionadas por conveniência duas escolas, uma particular (em uma região de Belo Horizonte considerada como área nobre) e uma pública estadual (em uma região considerada como economicamente carente em Contagem).

Foi elaborado um questionário especificamente para a pesquisa, consistindo de questóes de múltipla escolha, em que o indivíduo marcava apenas uma opção para cada questão. As perguntas foram assim descritas: 1) Dados sociodemográficos - tipo de escola (particular ou pública); idade, sexo, série escolar; 2) Classificação quanto ao nível socioeconômico, segundo 
a AssociaÇão Nacional das Empresas de Pesquisa (2008). Esse critério agrupa as pessoas nas classes A (alta), B, C, D e E (baixa), a partir da acumulação de bens materiais, das condições de moradia, número de empregados domésticos e do nível de escolaridade do chefe da família; 3) Classificação quanto ao EMC; nesse estudo fizemos uma adaptação ao modelo original, considerando uma sexta opção, a de recaída, em que o aluno já teria praticado uma AFL, mas, havia parado; 4) análise dos constructos da TCP: Atitude, subdividida em quatro tópicos (foi utilizada uma escala, variando de -3 "percepção negativa" $a+3$ "percepção positiva"), contendo questôes relativas a prática de atividades físicas, considerando-se o nível: a) nível de prazer com a prática dessas atividades; b) sabedoria ao se praticar essas atividades; c) utilidade dessa prática; e d) necessidade desse comportamento; Normas subjetivas (foi utilizada uma escala, variando de -3 "percepção negativa" a +3 "percepção positiva"); considerou: a) se pessoas que são importantes para o adolescente acreditam que ele deveria ou não praticar atividade física regular de lazer; b) se a pressão social é no sentido dele praticar ou não esse

\section{Resultados}

A TABELA 1 apresenta a distribuição dos alunos de acordo com os estágios de mudança de comportamento $\left(\mathrm{EMC}^{1}\right)$, os níveis socioeconômicos (NSE) e o sexo. Em geral, os participantes podem ser classificados como fisicamente inativos no lazer, representando $57 \%$ dos casos, composto em sua maioria das classes NSE C/D, e por moças.

Verificou-se a distribuição normal e a homogeneidade das médias das variáveis Med_A (relativa ao constructo Atitude), Med_N (relativa ao constructo Norma Subjetiva), e Med_C (relativa ao constructo controle), conforme os EMC. De acordo com o teste KolmogorovSmirnov, nenhuma das três variáveis da TCP pode ser considerada normal. No caso de Med_A (estatística K-S = 0,207 com 416 g.l, p < 0,001), Med_N (estatística $\mathrm{K}-\mathrm{S}=0,145$ com 416 g.l, $\mathrm{p}<0,001)$, Med_C (estatística K-S = 0,060 com 416 g.l, p = 0,001).

Apesar do não atendimento do pressuposto de normalidade, usou-se a ANOVA para comparar as médias da variável Med_A, conforme os EMC. A alternativa de usar um teste não-paramétrico (KruskalWallis) foi considerada. Obtiveram-se os mesmos resultados gerais, no sentido da detecção de alguma diferença entre pares de médias. No entanto, optou-se tipo de atividade; e c) se as pessoas, de modo geral, acreditam que ele deveria ou não praticar atividades físicas de lazer; Controle percebido - avaliação do controle percebido (foi utilizada uma escala, sendo o número 1 para a opção "discordo totalmente", até o número 7 "concordo totalmente"); considerou-se: a) a confiança do(a) adolescente que consegue praticar atividades físicas, caso esse seja seu desejo; b) o nível de dificuldade que ele(a) considera existir para a prática de tais atividades; c) se ele(a) tem o controle para escolher se vai ou não praticar essas atividades; e d) se realizar essas atividades depende apenas dele(a).

A amostragem inicial foi composta por 422 adolescentes; após a aplicação do questionário, seis alunos foram excluídos (representando 0,7\% da amostra), por apresentarem várias questões não respondidas - todos os alunos eram pertencentes às escolas públicas. Assim, a amostra final foi composta por 416 alunos (99,03\% da amostra inicial).

Para a análise dos dados, utilizaram-se os programas Excel ${ }^{\circledR} 2003$ e SPSS versão 13.0. Utilizaram-se estatísticas descritivas, técnicas de comparação de médias e de previsão, com nível de significância de 5\%.

pelo uso da ANOVA devido a sua robustez em relação à violação de normalidade e pela disponibilidade de testes "post hoc" para comparação de médias.

Comparadas as médias de Med_A dos EMC para as duas amostras juntas, encontrou-se $\mathrm{F}(5,387)=6,379$, com $\mathrm{p}<0,001$. Portanto, há diferença significativa entre as médias comparadas. Logo, foi necessário consultar os resultados dos testes "post hoc".

Após a análise do teste de post hoc (Bonferroni), apresentados na TABELA 2, que realiza as comparações da média da atitude quanto a prática de atividade física (Med_A) e sua interação com os EMC, observa-se que existe uma diferença estatisticamente significativa entre os grupos: a) Pré-contemplativo: ação, manutenção e recaída; b) Contemplativo: manutenção; c) Preparação: manutenção; d) Ação: contemplação; e) Manutenção: pré-contemplativo, contemplativo; f) Recaída: Pré-contemplativo. Ainda destaca-se que não foram observadas diferenças estatisticamente significativas entre médias de atitude (variável Med_A) nas demais comparações entre estágios, tomados dois a dois $(\mathrm{p}>0,05)$.

Para a análise da interação dos efeitos das variáveis EMC e NSE, fez-se a comparação de médias de 
MACIEL, M.G. \& VEIGA, R.T.

Med_A, usando-se a ANOVA com dois fatores fixos - EMC e NSE -, não se constatou diferenças significativas para o efeito principal NSE $(\mathrm{F}(1,381)$ $=2,194, \mathrm{p}=0,139$ com $\left.\eta^{2}{ }_{\text {parcial }}=0,006\right)$ e para $\mathrm{a}$ interação entre $\operatorname{EMC~e~} \operatorname{NSE}(\mathrm{F}(5,381)=1,154$, p = $\left.0,331, \operatorname{com} \eta_{\text {parcial }}^{2}=0,015\right)$. Detectou-se diferença estatisticamente significativa apenas para o efeito principal EMC $(\mathrm{F}(5,381)=6,638, \mathrm{p}<0,01, \mathrm{com}$ $\left.\eta_{\text {parcial }}^{2}=0,080\right)$.

Realizou-se então uma ANOVA com dois fatores, excluindo-se a interação EMC-NSE. O teste de Levene apresentou resultado não significativo $(\mathrm{F}(11$, $381)=0,968, p=0,475)$, indicando homogeneidade de variâncias.

TABELA 1 - Distribuição dos alunos de acordo com os EMC, sexo e NSE .

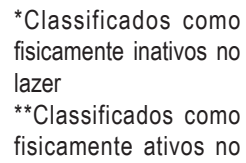

\begin{tabular}{|c|c|c|c|c|c|c|c|c|}
\hline \multirow{2}{*}{\multicolumn{2}{|c|}{ Variáveis }} & \multicolumn{6}{|c|}{ EMC } & \multirow{2}{*}{ Total (\%) } \\
\hline & & PC* & $\mathrm{C}^{*}$ & $\mathbf{P}^{*}$ & $\mathbf{A}^{* *}$ & $\mathbf{M}^{* *}$ & $\mathbf{R}^{*}$ & \\
\hline \multirow[b]{3}{*}{ NSE } & $\mathrm{A} / \mathrm{B}$ & 1,2 & 6,7 & 6,5 & 11,3 & 19,2 & 21,6 & 66,6 \\
\hline & $\mathrm{C} / \mathrm{D}$ & 1,9 & 5,5 & 5,8 & 4,8 & 7,7 & 7,7 & 33,4 \\
\hline & & 3,1 & 12,2 & 12,3 & 16,1 & 26,9 & 29,3 & \\
\hline \multirow{2}{*}{ SEXO } & $\mathrm{F}$ & 2,2 & 9,1 & 8,9 & 7,7 & 8,4 & 16,3 & 52,6 \\
\hline & M & 1 & 3,1 & 3,4 & 8,4 & 18,5 & 13 & 47,4 \\
\hline
\end{tabular}

TABELA 2 - Teste de Bonferroni Med_A.

*Nota: Nível de sig.0,05.

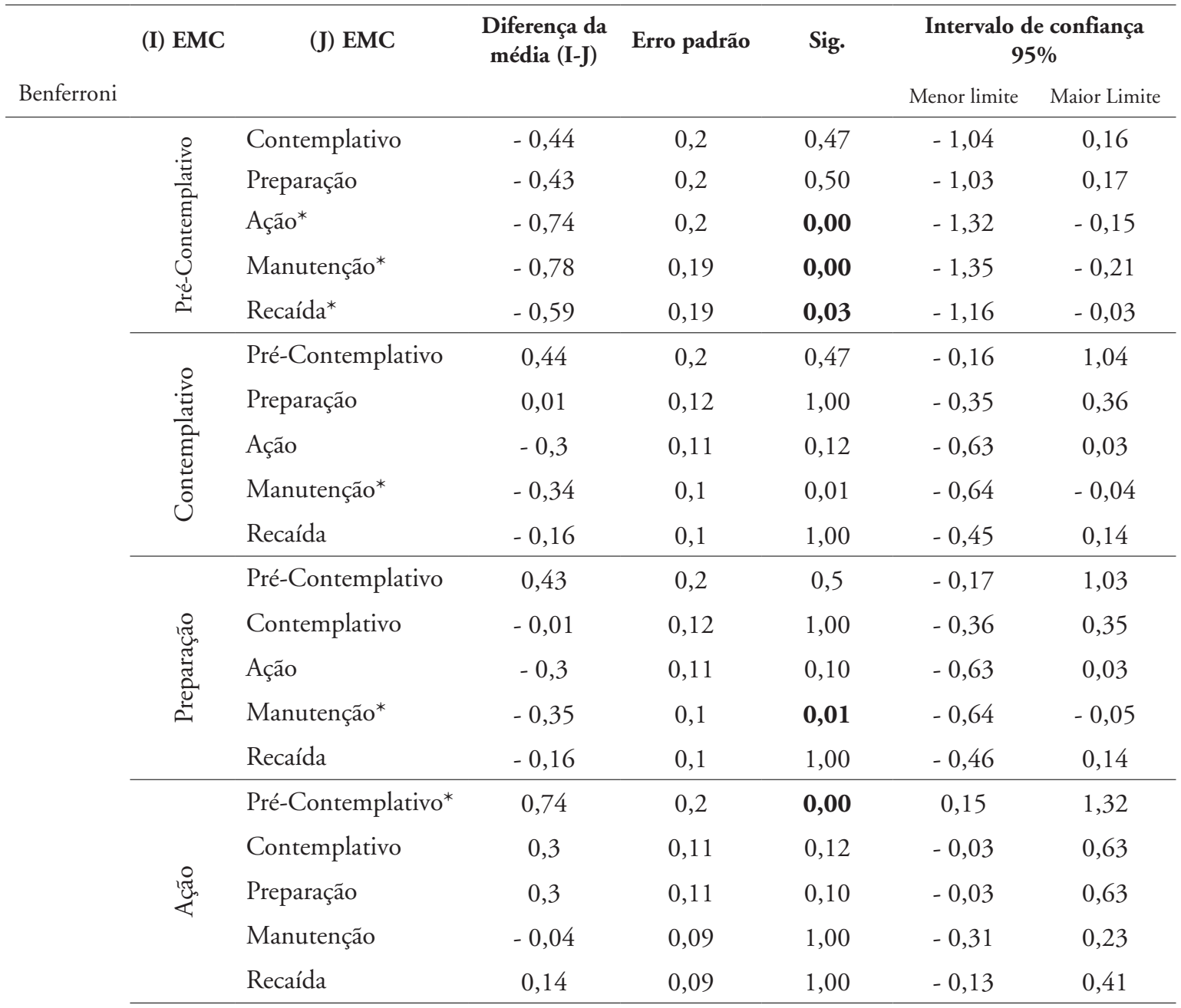

continua

708 • Rev. bras. Educ. Fís. Esporte, São Paulo, v.26, n.4, p.705-16, out./dez. 2012 
TABELA 2 - Teste de Bonferroni Med_A. (continuação).

\begin{tabular}{|c|c|c|c|c|c|c|c|}
\hline \multirow[b]{2}{*}{ Benferroni } & \multirow[t]{2}{*}{ (I) EMC } & \multirow[t]{2}{*}{ (J) EMC } & \multirow[t]{2}{*}{$\begin{array}{l}\text { Diferença da } \\
\text { média (I-J) }\end{array}$} & \multirow[t]{2}{*}{ Erro padrão } & \multirow[t]{2}{*}{ Sig. } & \multicolumn{2}{|c|}{$\begin{array}{c}\text { Intervalo de confiança } \\
95 \%\end{array}$} \\
\hline & & & & & & Menor limite & Maior Limite \\
\hline & \multirow{5}{*}{ 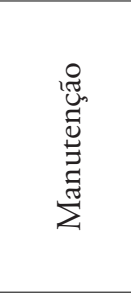 } & Pré-Contemplativo* & 0,78 & 0,19 & 0,00 & 0,21 & 1,35 \\
\hline & & Contemplativo* & 0,34 & 0,1 & 0,01 & 0,04 & 0,64 \\
\hline & & Preparação* & 0,35 & 0,1 & 0,01 & 0,05 & 0,64 \\
\hline & & Ação & 0,04 & 0,09 & 1,00 & $-0,23$ & 0,31 \\
\hline & & Recaída & 0,19 & 0,08 & 0,27 & $-0,05$ & 0,42 \\
\hline & \multirow{5}{*}{$\begin{array}{l}\underset{\widetilde{U}}{\tilde{Z}} \\
\mathscr{\mathscr { U }}\end{array}$} & Pré-Contemplativo* & 0,59 & 0,19 & $\mathbf{0 , 0 3}$ & 0,03 & 1,16 \\
\hline & & Contemplativo & 0,16 & 0,1 & 1,00 & $-0,14$ & 0,45 \\
\hline & & Preparação & 0,16 & 0,1 & 1,00 & $-0,14$ & 0,46 \\
\hline & & Ação & $-0,14$ & 0,09 & 1,00 & $-0,41$ & 0,13 \\
\hline & & Manutenção & $-0,19$ & 0,08 & 0,27 & $-0,42$ & 0,05 \\
\hline
\end{tabular}

TABELA 3 - Teste dos efeitos sem a interação das variáveis EMC e NSE.

\begin{tabular}{lcccccc}
\hline Fonte & $\begin{array}{c}\text { Soma dos quadrados } \\
\text { do Tipo III }\end{array}$ & g.l & $\begin{array}{c}\text { Quadrados } \\
\text { médios }\end{array}$ & F & Sig. & $\eta^{\mathbf{2}}$ \\
\hline Modelo corrigido & $11,018^{\mathrm{a}}$ & 6 & 1,836 & 5,399 & 0,00 & 0,077 \\
Intercepto & 1036,12 & 1 & 1036,12 & 3046,54 & 0,00 & 0,888 \\
EMC & 10,997 & 5 & 2,199 & 6,467 & $\mathbf{0 , 0 0}$ & $\mathbf{0 , 0 7 7}$ \\
NSE & 0,183 & 1 & 0,183 & 0,537 & 0,46 & 0,001 \\
Erro & 131,278 & 386 & 0,34 & & & \\
Total & 2388,25 & 393 & & & & \\
Total corrigido & 142,295 & 392 & & & & \\
\hline
\end{tabular}

$\mathrm{aR}^{2}=0,077\left(\mathrm{R}^{2}=0,063\right.$ ajustado).

Desconsiderando-se a interação entre as variáveis EMC e NSE (TABELA 3), no teste global da ANO$\mathrm{VA}$, verificou-se não haver significância no efeito da variável NSE $\left(\mathrm{F}(1,386)=0,537, \mathrm{p}=0,464 \mathrm{com} \eta_{\text {parcial }}^{2}\right.$ $=0,001)$, mas, somente entre os $\mathrm{EMC}, \operatorname{com~F}(5,386)$ $=6,467, \mathrm{p}<0,001, \mathrm{com} \eta^{2}$ parcial $=0,077$. Nessa análise constatou-se que o $\eta_{\text {parcial }}^{2}=0,077$ indica que $7,7 \%$ da variação de Med_A se deve à variável EMC.

Para a análise da interação dos efeitos das variáveis EMC e sexo, fez-se a comparação de médias de Med_A, usando-se a ANOVA com dois fatores fixos - EMC e sexo -, não se constatou diferenças estatisticamente significativas para o efeito principal sexo $(\mathrm{F}(1,381)=$ $1,8, \mathrm{p}=0,2 \operatorname{com} \eta^{2}$ parcial $\left.=0,005\right)$ e para a interação entre EMC e sexo $\left(F(5,381)=1,5, \mathrm{p}=0,2, \mathrm{com} \eta_{\text {parcial }}^{2}\right.$ $=0,019)$. Apenas foram detectadas diferenças estatisticamente significativa para o efeito principal EMC $\left(\mathrm{F}(5,381)=6,6, \mathrm{p}<0,01\right.$, com $\left.\eta^{2}{ }_{\text {parcial }}=0,080\right)$.

Realizou-se então uma ANOVA com dois fatores, excluindo-se a interação EMC-sexo. O teste de
Levene apresentou resultado não significativo $(\mathrm{F}(11$, $381)=1,631, p=0,088)$, indicando homogeneidade de variâncias.

Desconsiderando-se a interação entre as variáveis EMC e sexo, no teste global da ANOVA, verificouse não haver significância no efeito da variável sexo $\left(\mathrm{F}(1,386)=0,2, \mathrm{p}=0,694 \mathrm{com} \eta_{\text {parcial }}^{2}=0,001\right)$, mas, somente entre os $\mathrm{EMC}$, $\operatorname{com} \mathrm{F}(5,386)=6,1$, $\mathrm{p}<0,001$, com $\eta_{\text {parcial }}^{2}=0,073$. Nessa análise constatou-se que o $\eta_{\text {parcial }}{ }^{\text {parcial }}=0,073$, indica $7,37 \%$ da variação de Med_A se deve à variável EMC.

Para o cálculo da média das normas subjetivas (Med_N), utilizou-se o teste de Levene apresentou resultado não significativo $(\mathrm{F}(5,408)=0,766, \mathrm{p}=$ $0,575)$, indicando homogeneidade de variâncias, pressuposto da ANOVA.

Conforme o teste $\mathrm{F}$ da ANOVA verifica-se que o teste global de comparação de médias apresenta resultado estatisticamente não significativo, ou seja, não foram detectadas diferenças entre as médias 
$(\mathrm{F}(5,408)=1,349, \mathrm{p}=0,243)$, o que torna desnecessária a realização de testes "post-hoc".

Para a análise da interação dos efeitos das variáveis EMC e NSE, usou-se a comparação das médias de Med_N, usando-se a ANOVA com dois fatores fixos - EMC e NSE -, não se constatou diferenças estatisticamente significativas para o efeito principal NSE $\left(\mathrm{F}(1,402)=0,195, \mathrm{p}=0,659\right.$ com $\eta^{2}$ parcial $\left.<0,001\right)$ e para a interação entre $\operatorname{EMC~e~} \operatorname{NSE}(\mathrm{F}(5,402)=$ $0,843, \mathrm{p}=0,520$, com $\left.\eta_{\text {parcial }}^{2}=0,010\right)$; tampouco para o efeito principal EMC $(\mathrm{F}(5,402)=0,843, \mathrm{p}$ $<0,520$, com $\eta_{\text {parcial }}^{2}=0,010$ ).

Desconsiderando-se a interação entre as variáveis EMC e NSE, no teste global da ANOVA, verificouse não haver significância no efeito da variável $\operatorname{NSE}\left(\mathrm{F}(1,407)=2,360, \mathrm{p}=0,125 \mathrm{com} \eta^{2}\right.$ parcial $=$ $0,006)$, tampouco para o efeito principal EMC, com $\mathrm{F}(5,407)=1,021, \mathrm{p}=0,405, \mathrm{com} \eta_{\text {parcial }}^{2}=0,012$.

Para a análise da interação dos efeitos das variáveis EMC e sexo, fez-se a comparação de médias de Med_N, usando-se a ANOVA com dois fatores fixos - EMC e sexo -, não se constatou diferenças estatisticamente significativas para o efeito principal sexo $(\mathrm{F}(1,402)=$ $\left.0,327, \mathrm{p}=0,568 \mathrm{com} \eta_{\text {parcial }}^{2}=0,001\right)$ e para a interação entre $\mathrm{EMC}$ e sexo $(\mathrm{F}(5,402)=0,703, \mathrm{p}=0,580$, com $\eta^{2}$ arcial $\left.=0,009\right)$, tampouco o efeito principal EMC $\left(\mathrm{F}(5,402)=0,744 \mathrm{p}<0,51 \mathrm{com} \eta_{\text {parcial }}^{2}=0,009\right)$.

Desconsiderando-se a interação entre as variáveis EMC e sexo no teste global da ANOVA, verificouse não haver significância no efeito da variável sexo $\left(\mathrm{F}(1,407)=0,346, \mathrm{p}=0,557 \mathrm{com} \eta_{\text {parcial }}^{2}=0,006\right)$, tampouco para o efeito principal EMC, com $\mathrm{F}(5,407)=1,409, \mathrm{p}=0,220$, com $\eta_{\text {parcial }}^{2}=0,012$.

O cálculo das médias do controle percebido (Med_C), usou-se o teste de Levene, não foram encontradas diferenças estatisticamente significativas nas variâncias de Med_C para os estágios de mudança comportamental $(\mathrm{F}(5,410)=1,032$, $\mathrm{p}$ $=0,398)$. Atendido esse pressuposto, realizou-se a ANOVA comparando-se as médias do controle percebido (variável Med_C) nos estágios do Modelo Transteorético (variável EMC).Conforme resultados $\operatorname{de} \mathrm{F}(5,410)=8,438$, com $\mathrm{p}<0,001$, há diferença estatisticamente significativa entre algum par de médias comparadas, o que implica a necessidade de realizaçôes de testes "post hoc". Após a análise do teste de "post hoc" (Bonferroni), das comparações das médias do controle percebido quanto a prática de exercício físico (Med_C) e sua interação com os EMC, observa-se que existe uma diferença estatisticamente significativa entre os grupos: a) Pré-contemplativo: ação, manutenção, recaída; b)
Contemplativo: ação, manutenção; c) Preparação: ação, manutenção; d) Ação: pré-contemplativo, contemplativo, preparação; e) Manutenção: précontemplativo, contemplativo, preparação; f) Recaída: não houve interação. Ainda, destaca-se que não foram observadas diferenças estatisticamente significativas entre as demais interaçôes $(p>0,05)$.

Para a análise da interação dos efeitos das variáveis EMC e NSE, usou-se a comparação das médias de Med_C usando-se a ANOVA com dois fatores fixos - EMC e NSE -, não se constatou diferenças estatisticamente significativas para o efeito principal NSE $\left(\mathrm{F}(1,404)=1,469, \mathrm{p}=0,226 \mathrm{com} \eta_{\text {parcial }}^{2}=0,004\right)$ e para a interação entre EMC e NSE $(\mathrm{F}(5,404)=$ $\left.1,240, p=0,290, \operatorname{com} \eta^{2}{ }_{\text {parcial }}=0,015\right)$. Apenas foram detectadas diferenças para o efeito principal EMC $\left(\mathrm{F}(5,404)=7,041, \mathrm{p}<0,01, \mathrm{com} \eta^{2}\right.$ parcial $\left.=0,080\right)$.

Realizou-se então uma ANOVA com dois fatores excluindo-se a interação EMC-NSE. O teste de Levene apresentou resultado não significativo $(\mathrm{F}(11$, $404=1,457, \mathrm{p}=0,145)$, indicando homogeneidade de variâncias.

Desconsiderando-se a interação entre as variáveis EMC e NSE, no teste global da ANOVA, verificouse não haver significância no efeito da variável NSE $\left(\mathrm{F}(1,409)=0,379, \mathrm{p}=0,538 \mathrm{com} \eta_{\text {parcial }}^{2}=0,094\right)$, mas, somente entre os EMC, com $\mathrm{F}(5,409)=$ $8,475, \mathrm{p}<0,001$, com $\eta_{\text {parcial }}^{2}=0,094$. Nessa análise constatou-se que o $\eta_{\text {parcial }}^{2}=0,094$ indica $9,4 \%$ da variação de Med_C se deve à variável EMC.

Para a análise da interação dos efeitos das variáveis EMC e sexo, fez-se a comparação das médias de Med_C usando-se a ANOVA com dois fatores fixos - EMC e sexo -, não se constatou diferenças estatisticamente significativas para o efeito principal sexo $\left(\mathrm{F}(1,404)=0,213, \mathrm{p}=0,645 \mathrm{com} \eta^{2}{ }_{\text {parcial }}=0,001\right)$ e para a interação entre $\mathrm{EMC}$ e sexo $(\mathrm{F}(5,404)=$ $0,833, p=0,527$, com $\left.\eta_{\text {parcial }}^{2}=0,010\right)$. Apenas foram detectadas diferenças para o efeito principal EMC $\left(\mathrm{F}(5,404)=7,109, \mathrm{p}<0,01\right.$, com $\left.\eta_{\text {parcial }}^{2}=0,081\right)$.

Realizou-se então uma ANOVA com dois fatores, excluindo-se a interação EMC-sexo. $\mathrm{O}$ teste de Levene apresentou resultado não significativo $(\mathrm{F}(11$, $404)=0,960, p=0,483)$, indicando homogeneidade de variâncias.

Desconsiderando-se a interação entre as variáveis EMC e sexo, no teste global da ANOVA, verificouse haver significância no efeito da variável sexo $(\mathrm{F}(1$, 409) $=4,265, \mathrm{p}=0,04 \mathrm{com} \eta^{2}$ parcial $\left.=0,010\right)$; e também entre os $\mathrm{EMC}, \operatorname{com} \mathrm{F}(5,409)=6,495, \mathrm{p}<0,001$, $\operatorname{com} \eta^{2}$ parcial $=0,074$. Nessa análise constatou-se que o $\eta_{\text {parcial }}^{2}=0,074$ indica $7,4 \%$ da variação de Med_C se 
deve à variável $\mathrm{EMC} ; \eta_{\text {parcial }}^{2}=0,010$ indica que cerca de $10 \%$ da variação de Med_C se deve à variável sexo.

\section{Análise dos grupos classificados como fisicamente ativos e inativos no lazer através da regressão logística ordinal}

Para investigar a relação entre variáveis antecedentes da TCP, sexo e NSE com o nível de atividade física, criou-se uma variável binária dependente (ativo ou inativo), conforme os estágios do MT. Posteriormente, avaliaram-se modelos de regressão logística, para verificar se era possível prever se o indivíduo era ativo ou inativo a partir das variáveis explicativas da TCP, sexo e NSE. O objetivo da análise era avaliar quais variáveis são úteis para classificar corretamente o indivíduo como ativo ou inativo.

$\mathrm{Na}$ regressão logística as potenciais variáveis explicativas do modelo foram introduzidas sequencialmente: sexo, NSE, atitude, norma subjetiva e controle percebido. $\mathrm{O}$ objetivo final era avaliar o poder explicativo do modelo completo.

O teste de Hosmer e Lemeshow $\left(\chi^{2}(8)=6,779\right.$, $\mathrm{p}=0,561)$ apontou diferença estatisticamente significativa entre os grupos de ativos e inativos. O teste de Nagelkerke encontrou um $R^{2}=0,219$, o que quer dizer que, considerando-se as variáveis da TCP (atitude, norma subjetiva e controle percebido), NSE e sexo, o modelo logístico foi capaz de explicar $22 \%$ da variabilidade da variável-resposta, que representa o nível de atividade física, dicotomizado como ativo ou inativo. Para o grupo de ativos a capacidade de predição do modelo logístico foi de $32,7 \%$, e para o de inativos foi de $44,8 \%$; ou seja, após a desconsideração dos valores extremos citados, as variáveis explicativas utilizadas parecem mais efetivas para prever se o indivíduo será fisicamente inativo.

Teoricamente, não se pode assegurar que o modelo de regressão logística testado é completo porque o nível de atividade física também é influenciado por outras variáveis, tais como, fatores ambientais, equipamentos e espaços de lazer disponíveis para a prática de atividade física.

Aparentemente há um bom ajuste do modelo, baseando-se na inclusão dos antecedentes comportamentais como co-variáveis. Há diferença entre o logaritmo da razão de verossimilhança $(-2 L L)$, entre o modelo completo (seis parâmetros) e o modelo com apenas a constante (um parâmetro) é $\chi^{2}=70,061$, g.l. $=5$, valor-p $<0,001$. Assim, rejeita-se a hipótese nula de que todos os coeficientes do modelo são nulos. Ademais, o $\mathrm{R}^{2}$ de Nagelkerke, cujo limite teórico é
1, foi encontrado 0,219 para essa amostra, ou seja, cerca de $22 \%$ da variância da variável-resposta é explicada pelo modelo, o que é um valor razoável, porque medidas de síntese da regressão logística usualmente são bem menores do que os encontrados em regressão linear (NoRUsis, 2006).

Informaçôes mais detalhadas sobre o modelo completo são apresentadas na TABELA 5.

A equação para prever a probabilidade de ser ativo é:

$$
\operatorname{Prob}(\text { Ativo })=\frac{1}{1+\mathrm{e}^{-Z}}
$$

em que: $Z=-5,560+0,966$ (Sexo) $+0,023$ (NSE) + 0,641 (Atitude) + 0,012 (NS) +0,494 (CP).

Como os coeficientes das co-variáveis são positivos e não há interações, a razão das chances \{prob. (ser ativo) / prob (ser inativo)\} é maior do que 1, quando cada co-variável aumenta. Por exemplo, o aumento de uma unidade na escala de atitude corresponde ao aumento de 0,641 no logaritmo das chances, relacionado ao aumento da probabilidade de ser ativo.

Assim, por exemplo, um indivíduo de sexo feminino (sexo $=0)$, NSE A (NSE = 1), atitude $=3$, norma subjetiva $=2$, controle percebido $=4$ tem probabilidade de ser ativo de $\approx 0,167$, com $\log ($ chances $)=-0,701$. Em comparação a uma pessoa do sexo masculino (sexo=1) e os demais dados idênticos (NSE A, atitude $=3$ etc.), a probabilidade aumentaria para aproximadamente 0,343 , correspondente a $\log$ (chances $)=-0,281$.

A capacidade discriminativa do modelo pode ser apreciada no percentual de acerto de classificação do modelo em termos gerais: $68,4 \%$. Do ponto de vista de intervenção, a especificidade (proporção de inativos corretamente previstos $170 / 218 \cong$ $78 \%)$ pode ser tão importante quanto a sensibilidade (proporção de ativos corretamente previstos; $98 / 174 \cong 56,3 \%$ ), em função do objetivo geral de promover um estilo de vida saudável baseado nas atividades físicas.

Embora deva ser interpretada com cuidado, o valor da estatística Hosmer-Lemeshow $\left(\chi^{2}=6,779\right.$, g.l. $=8$, valor-p $=0,561)$, indica que o modelo está bem calibrado, com bom ajuste entre eventos observados e previstos, uma vez que o valor-p não significativo não leva à rejeição da hipótese nula de que não há diferença entre valores observados e previstos quando se comparam sub-amostras em que os casos totais são divididos em 10 grupos de tamanhos aproximadamente iguais (NORUSIS, 2006). 
MACIEL, M.G. \& VEIGA, R.T.

TABELA 4 - Resumo da análise sequencial do modelo logístico.

\begin{tabular}{|c|c|c|c|c|c|c|c|}
\hline \multirow{22}{*}{$\begin{array}{l}\text { Amostra com } 392 \text { casos: } \\
174 \text { ativos e } 218 \text { inativos; } \\
{ }^{* *} p<0,01 . \\
{ }^{*} p<0,05 \\
\text { NS: não significativo. }\end{array}$} & Passo & Covariáveis & B & $\begin{array}{c}\operatorname{Exp} \\
\text { (b) }\end{array}$ & $\begin{array}{c}\mathbf{R}^{2} \\
\text { Nagellkerke }\end{array}$ & $\begin{array}{c}\text { Percentual global } \\
\text { de classificação certa }\end{array}$ & $\begin{array}{c}\text { Percentual de acerto } \\
\text { de quem é ativo }\end{array}$ \\
\hline & 0 & Constante & $-0,225^{*}$ & 0,798 & & 55,6 & 0 \\
\hline & 1 & Constante & $-0,784^{* *}$ & 0,457 & 0,095 & 63,5 & 63,8 \\
\hline & & Sexo & $1,112^{* *}$ & 3,039 & & & \\
\hline & 2 & Constante & $-1,424^{* *}$ & 0,241 & 0,108 & 63,3 & 58,2 \\
\hline & & Sexo & $1,080^{* *}$ & 2,944 & & & \\
\hline & & NSE & $0,022^{*}$ & 1,023 & & & \\
\hline & 3 & Constante & $-3,284^{* *}$ & 0,037 & 0,162 & 65,6 & 54 \\
\hline & & Sexo & $1,070^{* *}$ & 2,916 & & & \\
\hline & & NSE & $0,023^{*}$ & 1,023 & & & \\
\hline & & Atitude & $0,768^{* *}$ & 2,154 & & & \\
\hline & 4 & Constante & $-3,279^{* *}$ & 0,038 & 0,162 & 65,6 & 54 \\
\hline & & Sexo & $1,070^{* *}$ & 2,914 & & & \\
\hline & & NSE & $0,023^{*}$ & 1,023 & & & \\
\hline & & Atitude & $0,7709^{* *}$ & 2,159 & & & \\
\hline & & Norma Subjetiva & $-0,007^{*}$ & 0,993 & & & \\
\hline & 5 & Constante & $-5,560^{* *}$ & 0,004 & 0,219 & 68,4 & 56,3 \\
\hline & & Sexo & $0,966^{* *}$ & 2,628 & & & \\
\hline & & NSE & $0,023^{*}$ & 1,023 & & & \\
\hline & & Atitude & $0,641^{* *}$ & 1,898 & & & \\
\hline & & Norma Subjetiva & $0,012 \mathrm{NS}$ & 1,012 & & & \\
\hline & & Controle Percebido & $0,494^{* *}$ & 1,639 & & & \\
\hline
\end{tabular}

TABELA 5 - Modelo logístico para prever o nível de atividade física.

Amostra com 392 casos: 174 ativos e 218 inativos. Sumário do modelo: $-2 L L=468,417$.

$\mathrm{R}^{2}$ de Coxe Snell $=0,164$; $\mathrm{R}^{2}$ de Nagelkerke $=0,219$. $\mathrm{NS}=$ norma subjetiva; $\mathrm{CP}=$ controle percebido.

\begin{tabular}{lcccccccc}
\hline Covariáveis & B & S.E. & Wald & d.f. & Sig. & Exp (B) & $\begin{array}{c}\text { Intervalo de confiança } \\
\text { 95\% (Chances) }\end{array}$ \\
\hline Constante & $-5,560$ & 0,835 & 44,384 & 1 & 0,00 & 0,004 & & 4,077 \\
Sexo & 0,966 & 0,224 & 18,591 & 1 & 0,00 & 2,628 & 1,694 & 1,047 \\
NSE & 0,023 & 0,012 & 3,988 & 1 & 0,045 & 1,023 & 1,000 & 2,802 \\
Atitude & 0,641 & 0,199 & 10,389 & 1 & 0,00 & 1,898 & 1,285 & 1,281 \\
\hline NS & 0,012 & 0,120 & 0,009 & 1 & $\mathbf{0 , 9 2}$ & 1,012 & 0,799 & 2,058 \\
\hline CP & 0,494 & 0,115 & 18,336 & 1 & $\mathbf{0 , 0 0}$ & 1,639 & 1,307 & \\
\hline
\end{tabular}

\section{Discussão}

O objetivo deste estudo foi investigar a intenção de mudança de comportamento de adolescentes de diferentes NSE para a prática de atividades físicas de lazer, adotando o Modelo Transteorético e a Teoria do Comportamento Planejado. Apesar das duas teorias adotadas nesse estudo serem as mais utilizadas para a investigação do fenômeno em questão, não foi encontrado na literatura nacional e/ou internacional, estudos que utilizassem a associação dessas duas teorias.

Considerando-se a análise das variâncias para os resultados apresentados dos antecedentes atitudinais 
da TCP, obtêm-se as seguintes conclusões para explicar a intenção de mudança do comportamento para a prática das atividades físicas na população estudada: 1) níveis de atitude variam conforme os EMC, porém não parecem ser significativamente influenciados pelas variáveis sexo e NSE; 2) níveis de normas subjetivas não diferem conforme o EMC, nem de acordo com as variáveis NSE e sexo; 3) níveis de controle percebido variam conforme as variáveis dos EMC e sexo, mas não parece ser influenciado pela variável NSE.

Esses dados levam a inferir que de acordo com as análises das amostras investigadas, atitude e controle percebido, podem ser considerados constructos mais relevantes para se promover uma intenção de mudança do comportamento para a prática das atividades físicas, podendo haver diferentes respostas conforme as variáveis sexo e os EMC. Assim, estratégias direcionadas para despertar um estilo de vida saudável devem promover uma atitude mais positiva em relação a prática de atividades físicas, bem como aumentar o controle percebido dos indivíduos, em termos de segurança, auto-eficácia e competência para que se sintam capazes de serem fisicamente ativos no lazer.

Segundo meta-análise realizada por Downs e HaUSEMBLAS (2005), a TCP teve o poder de predição entre $34 \%$ e $56 \%$, porém, os autores ressaltam a necessidade dos estudos serem mais esclarecedores quanto aos métodos empregados.

Neste trabalho, testou-se modelo de regressão logística binária, visando avaliar o poder das variáveis da TCP, acrescidas as variáveis sexo e NSE, para corretamente classificar os indivíduos como fisicamente ativos ou inativos no lazer, agrupados segundo os estágios do MT. O modelo logístico final obtido apresentou bom nível de ajuste estatístico, porém resultados de previsão apenas razoáveis. Nesse modelo todas as variáveis explicativas foram significativas $(\mathrm{p}<$ $0,05)$, com exceção da norma subjetiva.

O teste de Hosmer e Lemeshow $\left(\chi^{2}(8)=6,779\right.$, $\mathrm{p}=0,561)$ apontou diferença estatisticamente significativa entre os grupos de ativos e inativos. $\mathrm{O}$ teste de Nagelkerke encontrou um $R^{2}=0,219$, o que quer dizer que, no modelo logístico o conjunto de variáveis explicativas foi capaz de explicar $22 \%$ da variabilidade da variável resposta, que representa o nível de atividade física, dicotomizado como ativo ou inativo. No geral, a taxa de previsōes corretas foi razoável, aproximadamente $68,4 \%$ e prevendo apenas $56,3 \%$ dos indivíduos ativos.

Do ponto de vista de intervenção, a especificidade (proporção de inativos corretamente previstos $170 / 218 \cong 78 \%)$ pode ser tão importante quanto a sensibilidade (proporção de ativos corretamente previstos; $98 / 174 \cong 56,3 \%$ ), em função do objetivo geral de promover um estilo de vida saudável baseado nas atividades físicas. No entanto, verificou-se que o modelo logístico apresentou um desempenho melhor em termos de especificidade, o que aumenta seu valor potencial como meio de diagnosticar os indivíduos que tendem a ser fisicamente inativos.

No contexto pesquisado, sexo, atitude e controle percebido, são os fatores mais relevantes para classificar o nível de AFL, o que evidencia menor importância das variáveis sociais (NSE e norma subjetiva), pelo menos de acordo com o nível de precisão adotado na operacionalização desses constructos.

Diante dos resultados apresentados por esta pesquisa, as principais características da amostragem estudada são relatadas a seguir: a atitude está relacionada às crenças comportamentais que produzem uma avaliação (des)favorável em relação ao comportamento. Os resultados da pesquisa apontam uma associação entre a diferença das médias da atitude e alguns estágios do MT em relação a prática de atividades físicas (pré-contemplativo e ação; pré-contemplativo e manutenção; contemplativo e manutenção; preparação e manutenção). Esses resultados podem inferir que programas que visem mudar a atitude dos adolescentes em relação a essas práticas devem adotar estratégias diferenciadas para os EMC considerados.

Essas associaçóes mostram como as diferentes possibilidades de atitude interferem na intenção dos adolescentes efetivamente de exercerem o comportamento favorável para a prática das atividades físicas.

A pesquisa também mostrou que as variáveis sexo e NSE, não interferem nas atitudes dos adolescentes para explicar a intenção de mudança do comportamento para prática de atividades físicas, pelo menos na população estudada.

A norma subjetiva está relacionada às crenças normativas, que consideram as expectativas de terceiros e a pressão social perceptível. Os resultados da pesquisa apontam não existir uma diferença estatisticamente significativa das médias das normas subjetivas e alguns estágios do MT em relação a prática de atividades físicas.

Esses resultados sugerem que apesar da expectativa de terceiros e a pressão social poder alterar o comportamento dos adolescentes, confirmam a necessidade da adequação de estratégias distintas em relação àqueles que não têm a intenção de iniciar a prática de atividades físicas de lazer, ou que a abandonaram bem como daqueles que já estão engajados nessas práticas. 
Os dados da pesquisa ainda apontam que na população estudada, nem as variáveis NSE e o sexo, interferem no comportamento dos adolescentes em relação às expectativas e pressão social para a prática de atividades físicas.

O controle percebido está relacionado a fatores que podem impedir ou facilitar a adesão a um comportamento. Os resultados da pesquisa apontam uma associação entre a diferença das médias do controle percebido e alguns estágios do MT em relação a prática de atividades físicas (pré-contemplativo e ação; pré-contemplativo e manutenção; contemplativo e ação; contemplativo e manutenção; preparação e ação; preparação e manutenção).

Os resultados da pesquisa sugerem que há sensação do controle em relação a prática de atividades físicas, além da sensação de que executar tais atividades é algo fácil, pode alterar o comportamento dos adolescentes. Para os adolescentes não praticantes essas atividades, independente do estágio em que se encontram, os resultados podem inferir que as estratégias utilizadas em relação ao controle percebido devem ser diferentes, ou seja, fatores que podem impedir ou facilitar a adesão a um comportamento devem ser diferentes daqueles que já iniciaram a prática dessas atividades.

Além disso, segundo a amostragem estudada, nota-se que a variável NSE não interfere na percepção dos adolescentes de que a prática de atividades físicas é uma questão sob o seu controle; mas, a variável sexo interfere nessa percepção. Assim, pode-se apontar a necessidade de realização de campanhas de incentivo específicas diferenciadas de acordo com o sexo dos adolescentes, pois os rapazes consideram de forma diferente das moças esse fator.

Considerando os resultados apresentados, podese inferir que as variáveis normas subjetivas e nível socioeconômico, não são significativas para predizer a intenção de mudança do comportamento para a prática de atividades físicas na população estudada, ou seja, os adolescentes não consideram importantes a pressão/apoio social e as diferenças entre o poder aquisitivo para a prática dessas atividades.

No contexto pesquisado as variáveis sexo, atitude e controle percebido, são os fatores mais relevantes para classificar o nível de atividade física no lazer.

Conclui-se que há a necessidade de adaptação de campanhas para o incentivo da prática de AFL, adotando estratégias diferenciadas levando em consideração algumas características peculiares dessa população, bem como os fatores sócio-ambientais (barreiras e facilitadores), como o nível socioeconômico, sexo, ainda destaca-se a importância de se considerar os resultados aqui apontados relativos aos estágios do MT e os pressupostos da TCP para a definição dessas estratégias de incentivo.

\title{
Nota
}

1. Abreviações dos EMC - Pré-contemplativo: PC; Contemplativo: C; Preparação: P; Ação: A; Manutenção: M; Recaída: R.

\begin{abstract}
Intention of behavior changing in adolescents to practice leisure physical activities

The aim of this study was to investigate the intention of behavior changing in adolescents from different socioeconomic status (SES) to leisure physical activities, adopting the transtheoretical model and the Theory of Planned Behavior (TPB). We conducted a cross-sectional study involving 416 adolescents belonging to high schools in the cities of Belo Horizonte and Contagem / MG, with a mean age of 16.4 \pm 1.2 years. Analyses were performed using descriptive and parametric statistics (ANOVA and logistic regression). The results show that most of teenagers are inactive during leisure time, and the variables, sex, attitude and perceived control are the most relevant factors to rate the level of leisure physical activity, and social variables (socioeconomic status and standard subjective) are of less importance in predicting the intention to change behavior to practice these activities.
\end{abstract}

UnITERMs: Physical activity of leisure; Stage of behavior change; Theory of planned behavior. 


\section{Resumen}

Intención de cambio en el comportamiento de los adolescentes para la práctica de actividades físicas de ocio

El objetivo de este estudio fue investigar la intención de cambiar el comportamiento de los adolescentes de diferentes niveles socioeconómicos (NSE) para la práctica de actividades físicas de ocio, por la que se aprueba el modelo Transteoretico y la teoría de la Conducta Planificada (TCP). Se realizó un estudio transversal con la participación de 416 adolescentes, pertenecientes a las escuelas de enseñanza secundaria de las ciudades de Belo Horizonte y Contagem/MG, con una edad media de 16,4 $\pm 1,2$ años. Los análisis se realizaron mediante estadísticas descriptivas y paramétrico (ANOVA y regresión logistica), con $p<0,05$. Los resultados mostraron que la mayoría de los adolescentes e inactivo en su tiempo libre, y en cuanto a las variables, el género, la actitud y la percepción de control son los factores más relevantes para clasificar el nivel de actividad física de ocio, y que las variables sociales (nivel socio-económico y normas subjetivas) tiene poca importancia en la predicción de la intención de cambiar el comportamiento de la práctica de esas actividades.

Palabras clave: Actividad física de ocio; La etapa de cambio en el comportamiento; La teoría de la conducta planificada.

\section{Referências}

ASSOCIAÇÃO NACIONAL DE EMPRESAS DE PESQUISA (ANEP). Critério padrão de classificação econômica Brasil, 2008. São Paulo: ANEP, 2008.

AJZEN, I. From intentions to actions: a theory of planned behavior. In: KUHI, J.; BECKMAN, J. (Orgs.). Action control: from cognition to behavior. Heidelberg: Springer, 1985. p.11-39.

Perceived behavioral control, self-efficacy, locus of control, and the theory of planned behavior. Journal of Applied Social Psychology, Washington, v.32, p.1-20, 2002.

AJZEN, I.; FISHBEIN, M. Understanding attitudes and predicting social behavior. New Jersey: Prentice Hall, 1980. ARMITAGE, C.J. Can the theory of planned behavior predict the maintenance of physical activity? Health Psychology, Hillsdale, v.24, n.3, p.235-45, 2005.

BAGOZZI, R.P.; GÜRHAN-CANLI, Z.; PRIESTER, J.R. The social psychology of consumer behaviour. Buckingham: Open University, 2002.

BAUMAN, A.E.; SALLIS, J.F.; DZEWALTOWSKI, D.A.; OWEN, N. Toward a better understanding of the influences on physical activity: the role of determinants, correlates, causal variables, mediators, moderators, and confounders. American Journal Preventive Medicine, New York, v.23, p.5-14, 2002. Supplement 2.

BIDDLE, S.J.H.; NIGG, C.R. Theories of exercise behavior. International Journal of Sport Psychology, Rome, v.31, p.290-304, 2000.

BLANCHARD, C.; FISHER, J.; SPARLING, P.; NEHL, E.; RHODES, R.; COURNEYA, K.; BAKER, F. Understanding physical activity behavior in African American and Caucasian college students: an application of the theory planned behavior. Journal of American College Health, Washington, v.56, n.4, p.341-46, 2008.

CAMÕES, M.; LOPES, C. Fatores associados a atividade física na população portuguesa. Revista de Saúde Pública, São Paulo, v.42, n.2, p.208-16, 2008.

CHATZISARANTIS, N.I.D.; HAGGER, M.S.; BRICKELL, T. Using the construct of perceived autonomy support to understand social influence within the theory of planned behavior. Psychology of Sport and Exercise, Amsterdam, v.9, p.27-44, 2008. CHEUNG, S.Y. Exercise behavior for youth in Hong Kong. Research Quarterly for Exercise and Sport, Washington, p.A-89, 2007.

DOWNS, D.S.; HAUSENBLAS, A. Elicitation studies and the theory of planned behavior: a systematic review of exercise beliefs. Psychology of Sport and Exercise, Amsterdam, v.6, p.1-31, 2005.

DUMITH, S.C. Proposta de um modelo teórico para a adoção da prática de atividade física. Revista Brasileira de Atividade Física e Saúde, Londrina, v.13, n.2, p.52-62, 2008.

KRÜGER, H. Introdução à psicologia social. São Paulo: EPU, 1986. 
MACIEL, M.G. \& VEIGA, R.T.

MACIEL, M.G. Análise da intenção de mudança do comportamento em adolescentes para a prática de atividades físicas regulares de lazer. 2009. 126f. Dissertação (Mestrado em Lazer) - Escola de Educação Física, Fisioterapia e Terapia Ocupacional, Universidade Federal de Minas Gerais, Belo Horizonte, 2009.

NAHAS, M.V. Atividade física, saúde e qualidade de vida: conceitos e sugestôes para um estilo de vida saudável. 4. ed. rev. atual. Londrina: Midiograf, 2006.

NORUSIS, M. SPSS 15.0 statistical procedures companion. Chicago: Prentice-Hall, 2006.

PROCHASKA, J.O.; MARCUS, B. The transteoretical model: applications to exercise. In: DISHMAN, R.K. Advances in exercise adherence. Champaign: Human Kinetics, 1994. p.161-80.

; DiClemente C.C. Stages and processes of self-change in smoking: towards an integrative model of change. Journal

Consulting Clinical Psychology, Washington, v.51, p.390-95, 1983.

RHODES, R.E.; BLANCHARD, C.M.; COURNEYA, K.S.; PLOTNIKOFF, R.C. Identifying belief-based targets for the promotion of leisure-time walking. Health Education Behavior, Thousand Oaks, v.12, 2007.

SEABRA, A.F.; THOMIS, M.A.; ANJOS, L.A.; MAIA, J.A. Determinantes biológicos e sócio-culturais associados a prática de atividade física de adolescentes. Caderno de Saúde Pública, Rio de Janeiro, v.24, n.4, p.721-36, 2008.

WORLD HELATH ORGANIZATION. Global strategy on diet, physical activity and health. Genebra: WHO, 2004. Disponível:http://www.who.int/dietphysicalactivity/strategy?eb11344/en. Acesso em: 21 ago. 2004.

ZHANG, J.; MIDDLESTADT, S.E.; JI, C.Y. Psychosocial factors underlying physical activity. International Journal of Behavioral Nutrition and Physical Activity, London, v.4, p.38-48, 2007.

ENDEREÇO

Marcos Gonçalves Maciel Centro Desportivo

Universidade Federal de Ouro Preto

R. Paulo Magalhães Gomes, s/n - Campus Morro do Cruzeiro 35400-00o - Ouro Preto - MG - BRASIL e-mail: profmarcosmaciel@hotmail.com
Recebido para publicação: 06/05/2011

Revisado: 10/05/2012

Aceito: 25/05/2012 